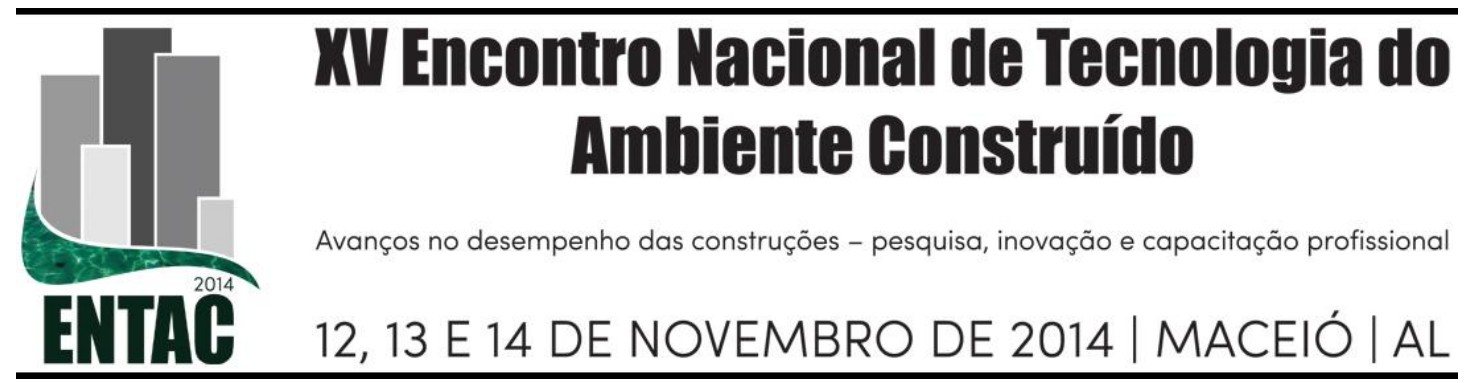

\title{
HABITAÇÃO DE INTERESSE SOCIAL EVOLUTIVA: ANÁLISE DE PROJETOS FLEXÍVEIS QUANTO À CONSTRUTIBILIDADE NO MOMENTO DE AMPLIAÇÃO
}

\author{
RAMÍREZ PEÑA, Arlene (1); BRANDÃO, Douglas Queiroz (2) \\ (1) Universidade Federal de Mato Grosso (UFMT), e-mail: arleneramirez@ hotmail.com \\ (2) Universidade Federal de Mato Grosso (UFMT), e-mail: dbrandao@ufmt.br
}

\begin{abstract}
RESUMO
A rigidez nas habitações e o fato de que a maioria seja construída de forma padronizada, ou seja, residências pensadas tão somente para atingir uma única configuração familiar tradicional, sem considerar as mudanças e a diversidade das necessidades dos usuários ao longo do tempo, constitui um problema vivenciado mundialmente. Há muitos anos vem-se discutindo o tema da flexibilidade espacial e das habitações evolutivas. Arquitetos e projetistas tem proposto alternativas tipológicas de habitações que se caracterizam pela flexibilidade e adaptabilidade de seus espaços. Embora as vantagens das habitações evolutivas sejam aparentemente maiores do que as desvantagens, a construção das mesmas não tem sido uma prática muito comum. O objetivo deste trabalho é analisar e comparar alguns casos de habitações evolutivas que se apresentam em destaque na literatura, estudando seu arranjo espacial e as estratégias utilizadas para alcançar a flexibilidade. Quatro casos foram selecionados e analisados quanto às direções e sentidos das possibilidades de ampliação, avaliando o grau de facilidade para se promover as expansões previstas, ou seja, se as construções futuras podem ser realizadas com rapidez e com menos perdas de materiais. Este artigo tem o propósito de despertar o interesse na temática das habitações evolutivas e flexíveis e difundir a continuação das boas práticas, além de motivar a criação de novas alternativas que possam ser aplicáveis ao setor da construção civil e proporcionar maior conforto aos usuários.
\end{abstract}

Palavras-chave: Habitação evolutiva, Flexibilidade espacial, Tipologias habitacionais.

\begin{abstract}
The rigidity in housing is a problem experienced worldwide. In fact the most residences are built on a standardized pattern designed solely to achieve a unique traditional family setting, without regard to changes and diversity of user needs over time. For many years the topic of flexibility and evolutionary dwellings has been discussed. Architects and designers have proposed typologies of housing that are characterized by the flexibility and adaptability of their spaces. Although the advantages of flexible housing are apparently greater than the disadvantages, these constructions are not a very common practice. The objective of this study is to analyse and compare some cases of evolutionary dwellings that appear highlighted in the literature, studying their typologies and strategies used to achieve flexibility. Four cases were selected and analysed regarding to the grow directions and also if the previewed expansions is easy to do during the construction moment, and if them can be doing quickly and with minimum rubble. This article is carried out in order to arouse interest in the subject of evolutionary and flexible housing and the continued spread of best practices and motivate the creation of new proposals that may be applicable in the construction sector and either offer comfort to users.
\end{abstract}

Keywords: Flexible housing, Space flexibility, House typologies. 


\section{INTRODUÇÃO}

O ser humano é um ser dinâmico, seus hábitos e atividades mudam ao longo do tempo, da mesma forma que mudam suas necessidades, entre elas as necessidades de distintos espaços na habitação. Morales e Alonso (2010) afirmam que a moradia é um processo que não termina em um determinado momento, mas, sim, vai se transformando e adotando diferentes configurações espaciais, dependendo das necessidades dos usuários ao longo da vida útil da moradia no tempo.

A moradia deve oferecer ao habitante conforto e prazer, deve ser um lugar do qual se sinta parte, e um espaço útil para a realização das distintas atividades de todos os ocupantes da habitação. A flexibilidade tem se convertido em um elemento primordial na hora de escolher uma moradia, devido à diversidade da família atual, os avanços tecnológicos e a variedade de usos que se está dando à habitação (trabalho, lazer, descanso etc.). A tendência é construir espaços que sejam adaptáveis a tais mudanças e necessidades.

Palermo e outros (2007) afirmam que estudos de compacidade revelam que a redução radical da área construída não acarreta redução de custos de construção na mesma proporção e que, por outro lado, o aumento planejado da área habitacional pode proporcionar maior satisfação aos usuários sem acarretar aumentos significativos nos custos da construção.

\subsection{Objetivos}

Os objetivos deste trabalho são:

- Apresentar uma breve revisão bibliográfica acerca dos conceitos principais relacionados à flexibilidade e habitação evolutiva.

- Estudar casos de habitações flexíveis destacados na literatura especializada, que possam ser considerados como exemplos de boas práticas, analisando suas tipologias e estratégias para alcançar a flexibilidade.

- Comparar os distintos casos, expondo vantagens e desvantagens construtivas.

O trabalho está estruturado na ordem dos objetivos apresentados, incluindo na última seção algumas conclusões que destacam os aspectos observados mais importantes.

\subsection{Método de pesquisa}

Este trabalho apresenta resultados parciais de um levantamento de projetos de habitação social evolutiva, a partir da literatura. Dentre mais de vinte casos encontrados, foram selecionadas 04 tipologias que fossem diferentes entre si, para analisar as formas de ampliação propostas. Os critérios de seleção foram: casas térreas de interesse social, com área a partir de $25 \mathrm{~m}^{2}$, planejadas para receber novos cômodos, ou seja, cuja planta trouxesse a proposta de casa embrião. Para cada planta foram desenhados esquemas para visualização dos espaços e análise dos sentidos das expansões previstas (frente, fundos, laterais), além da própria contagem do número de possibilidades.

\section{FLEXIBILIDADE NO PROJETO HABITACIONAL}

\subsection{A moradia e suas necessidades de modificação}

A moradia é mais que uma simples edificação para obter abrigo e segurança. Para Habraken, a moradia é a relação entre uma pessoa e seu entorno. Esta relação nasce nas 
ações cotidianas da vida e, portanto, está na essência de nossa existência. (HABRACKEN apud MORALES; ALONSO, 2010).

Segundo Morales e Alonso (2010), a moradia é comumente considerada como uma mercadoria e não como um direito ou um bem de uso. Porém, considerar a moradia como um processo e não um produto permite dar resposta às necessidades e possibilidades dos habitantes. Estes autores comentam que a moradia deve ser:

- Habitável: capaz de prover uma habitabilidade diversificada e adaptável que dê resposta à variedade de modos de vida atuais e futuros.

- Flexível: para poder se adaptar ao longo do seu ciclo de vida às mudanças das necessidades e requisitos dos moradores e do seu entorno.

- Acessível: no sentido de ser praticável para pessoas portadoras de necessidades especiais e também no sentido de ser acessível economicamente.

- Sustentável: deve reutilizar ao máximo os recursos existentes

Segundo Garcia e Haro (2010), os modelos arquitetônicos que uma vez são construídos e oferecidos à sociedade são automaticamente modificados por seus moradores para ajustar-se à realidade de suas necessidades, uma situação irracional e contraditória que não teria sentido em outras áreas como a indústria do automobilismo, eletrônica etc., onde o produto final se ajusta logicamente à demanda social.

As razões pelas quais o usuário deseja promover alterações em sua habitação são várias, sendo forte a ligação com fatores simbólicos e estéticos, como explica Brandão (2002). Estas alterações, de acordo com Reis (1995), estão ligadas principalmente a:

- Aspectos funcionais como disposição e tamanho das peças;

- Tamanho da moradia como um todo;

- Aspectos específicos ligados à privacidade visual e auditiva;

- Aspectos ligados a questões estéticas;

- Aspectos ligados a questões de personalização e definição do território;

- Alterações no tamanho da família, nível econômico e educacional;

- Aspectos de outra natureza, por exemplo, a necessidade de criar um abrigo para o carro ou ligados ao lazer, como a criação de uma churrasqueira.

Geralmente nas habitações a posição de elementos como esquadrias, áreas molhadas, telhados e outros espaços dificultam ou aumentam os custos de modificações e ampliações se estas não foram projetadas para evoluir.

Para Paiva (2002), a necessidade de diversidade habitacional tem cada vez maior importância, devido:

- Ao grande desenvolvimento tecnológico e o gosto pela novidade;

- A maior diversidade social e necessidade de satisfação pessoal;

- A necessidade psicológica de diversidade cultural e de expressão individual.

Paiva (2002) também inclui como motivos fundamentais para a criação de habitações flexíveis: a mobilidade da população, a mudança da composição social e as dinâmicas familiares.

Garcia e Haro (2010) explicam que as famílias passam por diversas fases ao longo do seu ciclo de vida e que nestas fases varia o tamanho, as relações interpessoais, as posições, os objetos e as necessidades de alojamento, equipamentos e serviços. Quando a habitação não tem a capacidade de se adaptar a tais mudanças, a família é que tem que se adaptar aos espaços que, em muitas vezes, não se ajustam a suas necessidades. 


\subsection{Habitação evolutiva e flexibilidade}

A habitação tem hoje uma maior necessidade para se adaptar às diferentes exigências funcionais. Não deve ser uma edificação rígida e estática que não aceite mudanças ou ampliações. Segundo Friedman (1997), a casa deve ser projetada para ser adaptável ao mercado de usuários desconhecidos, de maneira que no estágio de pré-ocupação o construtor possa modificar a moradia às necessidades de diferentes tipos de famílias e que no estágio de pós-ocupação a casa responda às necessidades de mudança espacial, e seja facilmente adaptável às necessidades de novas famílias quando os primeiros se mudarem.

Uma habitação é considerada evolutiva ou polivalente quando dada a maneira como foram concebidos os seus espaços, permite alterar os usos dentro dela, ocupá-la de maneiras variadas, distribuindo as funções diferentemente (ROSSO, 1980).

O termo flexibilidade é muito amplo e abarca uma grande quantidade de variáveis e se presta a um grande número de classificações. Paricio (1998 apud PAIVA, 2002) define a flexibilidade como qualquer disposição construtiva ou formal, que permita determinada diversidade nas suas formas de ocupação. A flexibilidade na habitação permite que a mesma seja adaptável às mudanças de necessidades e requisitos dos usuários e do seu entorno ao longo do seu ciclo de vida.

Brandão (2002), a partir de um estudo sobre as formas de aplicação da flexibilidade arquitetônica sintetiza seis formas principais de aplicação da flexibilidade:

- Diversidade tipológica: quando se explora apenas a variabilidade, sem possibilidade de modificação;

- Flexibilidade propriamente dita: quando se pode gerar mais de um arranjo, obtendo variabilidade por meio de construção;

- Adaptabilidade: quando se pode obter a alternância ou a sobreposição de funções nos ambientes, sem construção, seja pela neutralidade do ambiente, seja pelo uso de elementos móveis ou facilmente removíveis. Algumas características facilitadoras: simplicidade, ambiguidade, espaço extra, neutralidade, baixa hierarquia, e, ainda, a multifuncionalidade ou agregação de funções. A neutralidade, de acordo com Albers, Henz e Jacob (1989), ocorre quando se definem peças com nenhuma utilização previamente especificada, ou seja, neutras, que podem ser utilizadas por distintos habitantes para diferentes atividades;

- Ampliabilidade: ocorre quando há possibilidade de adicionar novos ambientes ou cômodos à edificação. A aplicação deste princípio está vinculada às restrições de ocupação do solo. A adição de um ou mais quartos implica no estudo de uma disposição inicial que permita uma integração razoável no projeto final (ROSSO, 1980). Pode ser interna ou externa;

- Junção/desmembramento: quando a habitação como um todo pode ser dividida em duas ou, de forma contrária, quando duas habitações contíguas forem agrupadas, formando uma só. Tais características não são excludentes, ou seja, podem ser combinadas em um mesmo projeto para alcançar uma maior flexibilidade.

Rabeneck, Sheppard e Town (1974 apud BRANDÃO, 2002) relacionam uma serie de componentes básicos para que um esquema flexível possa ser obtido. Estes são:

- Divisórias internas não portantes e removíveis;

- Ausência de colunas ou, grandes vãos entre elementos e vedos portantes; 
- Instalações, tubulações e acessórios desvinculados da obra bruta, evitando embuti-los na alvenaria;

- Marginalização da área úmida e das instalações de serviços em relação à seca;

- Localização das portas e das janelas de maneira a permitir mudança de posição;

- Utilização de formas geométricas simples nos quartos;

- A não utilização, da locação central dos aparelhos de iluminação.

\section{ESTUDO DE CASOS}

\begin{tabular}{|c|c|c|}
\hline Projeto: & \multicolumn{2}{|c|}{ Projeto Moradia, Programa Habitare, (Projeto XIV) } \\
\hline Localização: & \multicolumn{1}{|c|}{ CEFETMT/UFMT } \\
\hline Projetistas: & $\begin{array}{l}\text { Cposta Programa Habitare, Barra do Bugres, Mato Grosso (2003-2006) } \\
\text { sala-cozinha-banheiro. Propõe o mínimo para se morar, } \\
\text { ou seja, reunindo toda a parte hidráulica da casa, porém } \\
\text { de forma ainda precária e provisória em termos de } \\
\text { moradia, já que os moradores utilizariam a sala de estar } \\
\text { como dormitório até que se possa construir um ou mais } \\
\text { dormitórios. } \\
\text { A Casa XIV adota um sistema de crescimento radial, } \\
\text { permitindo ampliações para frente, para os fundos e para } \\
\text { uma das laterais da casa, oferecendo, assim, maior } \\
\text { versatilidade. Os dormitórios podem ser adicionados ao } \\
\text { núcleo de várias formas: frente e fundos, frente e lateral, } \\
\text { ou, lateral e fundos. O quarto dos fundos possui duas } \\
\text { opções de implantação, alinhado ou não com relação ao } \\
\text { quarto da frente. Também a alteração da fachada é livre, } \\
\text { devido às possibilidades de expansão para frente. }\end{array}$ \\
\hline Fonte: Rodrigues; Brandão, (2005)
\end{tabular}

\begin{tabular}{|c|c|}
\hline Projeto: & \multicolumn{1}{|c|}{ Casa do Residencial Wilton Lira } \\
\hline Localização: & \multicolumn{1}{|c|}{ Caruaru, PE } \\
\hline Projetistas: & $\begin{array}{l}\text { Pablo Patrioca. Jirau Arquitetura } \\
\text { Eeferência modeto de habitação social que se caracteriza pela } \\
\text { fachadas e pelo bom aproveitamento do terreno, } \\
\text { formando um conjunto organizado por quadras em lotes. } \\
\text { A planta do projeto é composta por sala, cozinha, } \\
\text { banheiro e dois quartos à frente da casa, organizados em } \\
\text { forma de “S”. O projeto prevê o aumento de um quarto e } \\
\text { um banheiro que se abrem para o quintal, sem afetar a } \\
\text { estrutura e a estética do projeto original, dando } \\
\text { importância à conservação da fachada. Para manter a } \\
\text { referência do conceito habitual de casa, o telhado frontal } \\
\text { é inclinado e de telha cerâmica com um amplo beiral. }\end{array}$ \\
\hline Fonte: Frajndlich (2012)
\end{tabular}




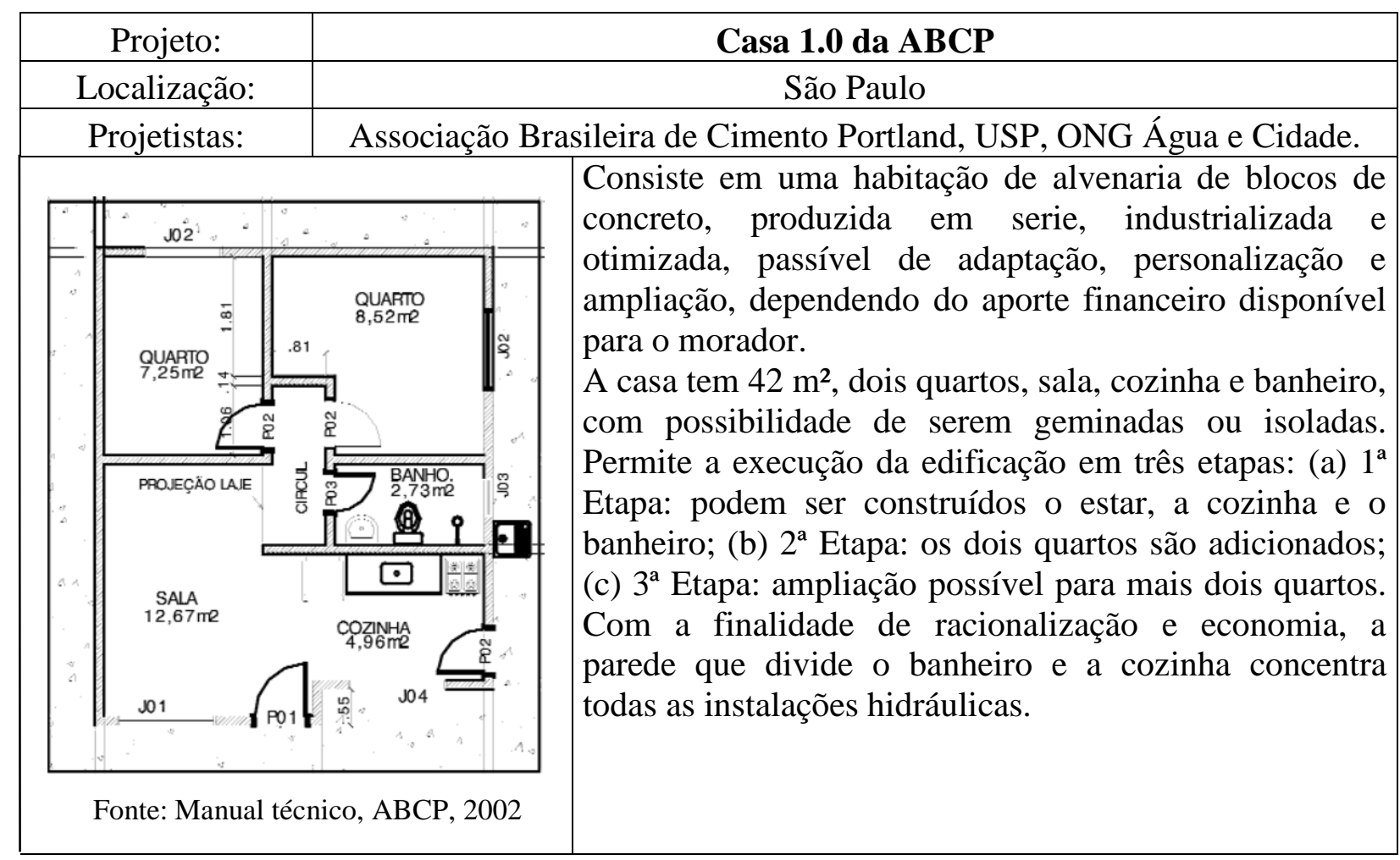

\begin{tabular}{|c|c|}
\hline Projeto: & Casa de madeira que pode ser ampliada para o lado e para frente \\
\hline Localização: & \multicolumn{1}{|c|}{ Desconhecido } \\
\hline Projetistas: & $\begin{array}{l}\text { Exemplo de uma casa construída em madeira que utiliza } \\
\text { a estratégia de divisórias desmontáveis ou móveis para } \\
\text { inserir flexibilidade. A casa possui uma divisória entre a } \\
\text { copa-cozinha e a sala de estar que também pode ser } \\
\text { utilizada como quarto. Com a ampliação, a divisória } \\
\text { pode ser desmontada e recolocada em outra posição na } \\
\text { casa. Esta estratégia permite que os arranjos possam ser } \\
\text { modificados de uma maneira simples e rápida. A } \\
\text { utilização de móveis e componentes fixos deve ser } \\
\text { evitada o máximo possível para assim oferecer uma } \\
\text { maior diversidade de arranjos e uso dos espaços } \\
\text { possíveis dependendo das necessidades. }\end{array}$ \\
Fonte: Oliveira (2003) \\
(2006).
\end{tabular}

\footnotetext{
${ }^{1}$ OLIVEIRA, C. F. Autoconstrução em madeira. Dissertação de Mestrado. Programa de Pós-graduação em Arquitetura da Escola de Engenharia de São Carlos, USP, São Carlos-SP, 2003.
} 


\section{ANALISE COMPARATIVA DOS CASOS}

Sem levar em conta as áreas dos cômodos, nem tampouco o layout interno, mas tão somente a simples agregação das peças (cômodos), procurou-se analisar os casos, buscando uma melhor caracterização da ampliabilidade proposta em cada um, e, consequentemente, comparando os projetos.

No quadro 1 é observada a opção de embrião sala-cozinha-banheiro, observando que somente a Casa 1.0 da ABCP e a Casa XIV do Projeto Moradia apresentam esta alternativa. Estes dois projetos utilizam três portas e cozinha integrada à sala de estar que funciona também como espaço de refeições. A diferença entre os dois modelos de embrião está no planejamento dos sentidos das ampliações: enquanto a Casa 1.0 permite expandir somente para os fundos, a Casa XIV adota um sistema radial, permitindo ampliações para frente, para os fundos e para uma das laterais da casa.

Quadro 1- Proposta de embrião sala-cozinha-banheiro nos casos estudados.

\begin{tabular}{|c|c|c|c|}
\hline $\begin{array}{c}\text { Residencial Wilton Lira, } \\
\text { Caruaru, PE }\end{array}$ & Casa 1.0 da ABCP & Casa de madeira & $\begin{array}{c}\text { Casa XIV, Projeto Moradia, } \\
\text { Mato Grosso }\end{array}$ \\
\hline $\begin{array}{c}\text { Não foi proposto embrião sala- } \\
\text { cozinha-banheiro }\end{array}$ & $\begin{array}{c}\text { Fão foi proposto } \\
\text { Frente }\end{array}$ & $\begin{array}{c}\text { Fanheiro } \\
\text { bano sala-cozinha- }\end{array}$ \\
\hline
\end{tabular}

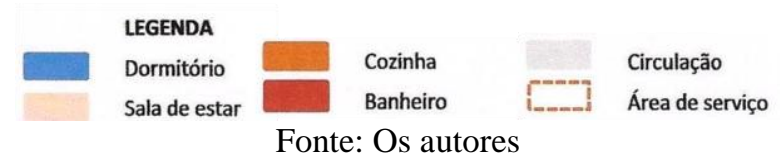

No quadro 2 a comparação é feita com um programa de dois dormitórios em cada casa. Como já dito, a casa em Caruaru, Pernambuco, foi idealizada desde o início com dois dormitórios à frente da casa. $\mathrm{Na}$ casa de madeira os dois primeiros dormitórios ficam aos fundos, o mesmo acontecendo com a Casa 1.0. Já a Casa XIV possibilita quartos na frente, nos fundos e em um dos lados.

Quadro 2- Proposta de casa com dois dormitórios nos casos estudados.

\begin{tabular}{|c|c|c|c|}
\hline $\begin{array}{c}\text { Residencial Wilton Lira, } \\
\text { Caruaru, } \mathrm{PE}\end{array}$ & Casa 1.0 da ABCP & Casa de madeira & $\begin{array}{c}\text { Casa XIV, Projeto Moradia, } \\
\text { Mato Grosso }\end{array}$ \\
\hline Frente & & & \\
\hline
\end{tabular}

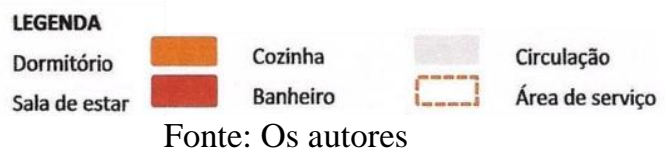


Por fim, no quadro 3, é apresentada a análise da expansão das casas com o programa de três dormitórios. No Residencial Wilton Lira, só há uma posição para o terceiro dormitório. Nos demais casos há mais de uma opção para locação do terceiro dormitório. Na casa de madeira a previsão do novo quarto pode ser contígua aos demais, porém o espaço de aumento da sala poderia ser usado também com a função de dormitório. Na Casa 1.0, há duas opções para a locação do terceiro dormitório, podendo se escolher o lado direito ou esquerdo da casa. E, por fim, a Casa XIV é a mais versátil, já que o terceiro dormitório pode ser projetado em vários locais, na frente nos fundos e na lateral.

Quadro 3- Proposta de casa com três dormitórios nos casos estudados.

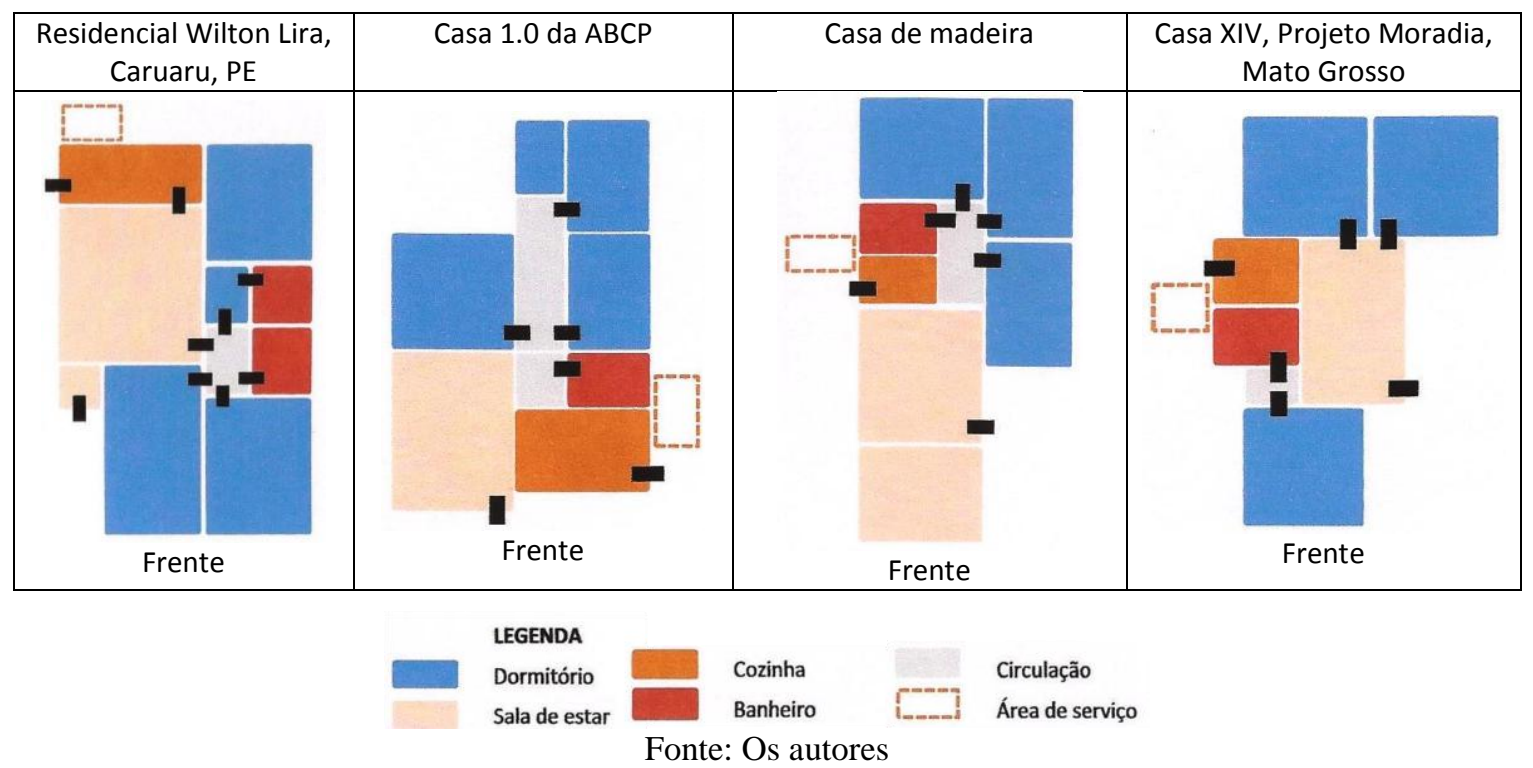

O quadro 4 sintetiza as possibilidades de ampliação no que se refere aos sentidos da expansão proposta. Observa-se que os quatro casos estudados apresentam-se diferentes entre si, o que demonstra o grande potencial de possibilidades para se promover habitações evolutivas. A casa de Caruaru, Pernambuco, foi a única a apresentar a proposta de suíte, com a inclusão de um segundo banheiro, sendo positivo o fato dos dois banheiros serem contíguos para aproveitamento de pontos hidráulicos.

Sobre a observação dos sentidos das ampliações é importante ressaltar que tanto a Casa do Residencial Wilton Lira (Caruaru, PE) quanto a Casa 1.0, preservam a fachada frontal, visto que as ampliações podem ser feitas somente para os fundos. Com isso a composição arquitetônica é mantida já que as expansões não são visíveis para quem está na rua. Pode ser considerado um fato positivo ou negativo, dependendo do ponto de vista: ou preservar a fachada original ou poder alterar a fachada. Nos casos da casa de madeira e Casa XIV, a alteração da fachada é livre, sendo as possibilidades de expansão para frente. Aqui há também o dilema: por um lado há liberdade de compor formatos variados de fachada, por outro lado, caso não haja orientação técnica qualificada (sobretudo do trabalho de arquitetos), fachadas muito ruins em termos estéticos podem ser construídas. Entende-se que, embora a habitação seja flexível, diretrizes e possibilidades de ampliações que foram pensadas no momento do projeto da habitação devem ser providas ao usuário. 
Quadro 4- Casos estudados e as possibilidades de ampliação em termos de sentido da expansão.

\begin{tabular}{|l|c|c|c|}
\hline & Frente & Fundos & Lateral \\
\hline Residencial Wilton Lira, Caruaru, PE & & $\mathbf{x}$ & \\
\hline Casa 1.0 da ABCP & & $\mathbf{x}$ & \\
\hline Casa de madeira & $\mathbf{x}$ & & $\mathbf{x}$ \\
\hline Casa XIV, Projeto Moradia, Mato Grosso & $\mathbf{x}$ & $\mathbf{x}$ & $\mathbf{x}$ \\
\hline
\end{tabular}

Fonte: os autores

\section{CONSIDERAÇÕES FINAIS}

Viu-se que, em apenas quatro casos analisados, varias alternativas podem ser adotadas para promover a ampliabilidade. Geometricamente quatro possibilidades foram observadas quanto ao sentido de crescimento da casa: somente para os fundos; para os fundos e para a lateral; para frente e para a lateral; radialmente, ou seja, para frente, fundos e lateral.

Uma estratégia de suma importância é fixar painéis-janela que tenham a dimensão de uma porta normal, posicionadas estrategicamente para funcionarem futuramente como porta nas ampliações. Os painéis-janela retirados seriam utilizados nos novos cômodos, ou seja, não havendo perda de materiais. Esta proposta foi apresentada pela Casa XIV. Há, obviamente, um custo inicial para prover estes painéis-janelas, mas se justifica na medida em que torna prática e rápida qualquer ampliação, além de dirigir o usuário para as melhores possibilidades (que devem estar no manual da casa).

Como exposto neste trabalho, pelo menos alguma estratégia de flexibilidade é necessária, devendo ser concebida no projeto inicial e adotada nos empreendimentos sociais. Possibilitar adaptar a casa é importante uma vez que, como já comentado, há muitos perfis de usuários, várias configurações familiares na atualidade, além das próprias mudanças destes usuários ao longo do tempo. Trata-se, em resumo, de evitar que os espaços se tornem obsoletos e disfuncionais.

Seria muito adequado promover habitações planejadas a partir da diversidade e da individualidade, mais que a partir da homogeneidade e da coletividade, tendo em consideração uma serie de fatores que permitem alcançar a flexibilidade sem necessariamente implicar em elevação dos custos. Existe grande número de estratégias para inserir flexibilidade aos projetos, não se limitando à simples ampliação ou adição de espaços. É possível obter flexibilidade também mediante a criação de espaços neutros, mediante a boa localização dos espaços, com a utilização de divisórias e móveis, a utilização de corretos sistemas de iluminação e ventilação entre outras.

E, outro aspecto que vale considerar, refere-se às fachadas, pois as habitações flexíveis e evolutivas podem enriquecer os conjuntos habitacionais, na medida em que se promove a diversidade, a liberdade de gerar casas personalizadas, em oposição aos conjuntos monótonos, com grande número de unidades iguais, típicos dos programas em vigor no país. Nos manuais das casas evolutivas podem ser propostas soluções de qualidade estética para as várias possibilidades de fachada.

Sugere-se para trabalhos futuros continuar aprofundando os estudos sobre flexibilidade e habitação evolutiva, avaliando e propondo estratégias e sistemas que proporcionem projetos de qualidade arquitetônica, mas, principalmente, que estas propostas sejam postas em prática pelos programas habitacionais e não fiquem só como propostas. Tratase de um desafio frente às políticas e programas de HIS vigentes, normalmente desprovidos de qualidade de projeto e com foco restrito à produtividade e mercado. 


\section{AGRADECIMENTOS}

A CAPES pela concessão de bolsa de mestrado, condição essencial para realização deste estudo.

\section{REFERÊNCIAS}

ALBERS, M.; HENZ, A.; JACOB, U. Wohnformund Wohnungs form.Wohnungen fürunters chiedliche Haushaltformen [Apartamentos para vários tipos de organização familiar]. Werk, Bauenund Wohnen, Zuerich, v.76/43, n.5, p.60-73, 1989.

ASSOCIAÇÃO BRASILEIRA DE CIMENTO PORTLAND (ABCP) Manual técnico para implementação - Habitação 1.0: Bairro Saudável. População Saudável. São Paulo: ABCP - Associação Brasileira de Cimento Portland, set./2002. 88 p.

BRANDÃO, D. Q. Diversidade e potencial de flexibilidade de arranjos espaciais de apartamentos: uma análise do produto imobiliário brasileiro. 2002. $443 \mathrm{f}$. Tese (Doutorado em Engenharia de Produção) - Programa de Pós-Graduação em Engenharia de Produção, Universidade Federal de Santa Catarina, Florianópolis.

Habitação social evolutiva: aspectos construtivos, diretrizes para projetos e proposição de arranjos espaciais flexíveis. Cuiabá: CEFETMT, 2006. Manual técnico.

FRAJNDLICH, R. U. Modernas referências. Arquitetura e Urbanismo. São Paulo, Pini, n. 224, nov.2012, p. 32-37.

FRIEDMAN, A. Design for flexibility and affordability: learning from the post-war home. Journal of Architectural and Planning Research, Chicago, v.14, n.2, p.150170, Summer, 1997.

GARCIA, T. G.; HARO, A. A. G. Espacio doméstico y nuevos usuarios: hábitos y transformaciones en la vivienda. Dirección General de Arquitectura y Vivienda .Junta de Andalucía. Sevilla, Junio de 2010.

MORALES, E. S.; ALONSO R. M. Proyecto de investigación "casa más o menos: la vivienda como proceso". 2010, La Panadería, Arquitectura y Diseño S.L.P, Consejería de Vivienda y Ordenación del Territorio Junta de Andalucía, Andalucía, 2010.

PAIVA, A. L. S. A. Habitação flexível: análise de conceitos e soluções. 2002. Lisboa. Dissertação (Mestrado em Arquitetura) - Faculdade de Arquitetura, Universidade Técnica de Lisboa, Lisboa, 2002.

PALERMO, C.; MORAIS, G.; COSTA, M.; FELIPE, C. Habitação Social: Uma visão projetual. In: Colóquio de Pesquisas em Habitação, 4, 2007. Anais... Disponível em: <http://www.mom.arq.ufmg.br/mom/coloquiomom/comunicações/palermo.pdf >. Acesso em abril2013.

ROSSO, T. Racionalização da construção. São Paulo: USP/FAU, 1980. 300p. 